\title{
Kriminologischer Beitrag
}

\section{"Werther-Effekt" und „Bullycide"-Medienkonsum, Cybermobbing und Suizidalität von Kindern und Jugendlichen}

\author{
Marleen Gräber' • Barbara Horten'
}

Eingegangen: 12. August 2020 / Angenommen: 27. August 2020 / Online publiziert: 28. September 2020

(c) Der/die Autor(en) 2020

Der vorliegende Beitrag beleuchtet den Zusammenhang zwischen dem Konsum und der Nutzung digitaler Medien und aggressivem und selbstschädigendem Verhalten von Kindern und Jugendlichen. Die Schädigung anderer Kinder und Jugendlicher durch TäterInnen in ähnlichem Alter mittels digitaler Medien wird unter den Begriffen des sog. Cybermobbing bzw. Cyberbullying. (nach dem engl. to bully: schikanieren) subsumiert, das in den letzten Jahren vermehrt auftritt. Back (2020) klassifiziert Cybermobbing, angelehnt an Smith et al. (2008), als „(...) aggressiver, intentionaler Akt, welcher von Gruppen oder Individuen wiederholt über längere Zeit gegen ein wehrloses Opfer durchgeführt wird, durch den Gebrauch elektronischer Formen der Kommunikation" (Hervorhebungen im Original) (S. 61). Die schwerste Folge von Mobbing-Attacken besteht in dem Suizid des Opfers.

Selbsttötungen unter Jugendlichen im Alter von 15 bis 20 Jahren sind nach dem Unfalltod die zweithäufigste Todesursache in dieser Altersgruppe (Radeloff et al. 2012, S. 263). Nach Back (2020), die sich auf Angaben der Centers for Disease Control und Prevention (2011) bezieht, sind Selbsttötungen in der Altersgruppe der 10- bis 14-Jährigen die dritthäufigste Todesursache. Das Statistische Bundesamt verzeichnete im Jahr 201813 Suizide in der Gruppe der 10bis 15-Jährigen ( 8 männlich und 5 weiblich) und 179 Suizide in der Gruppe der 15- bis 20-Jährigen (132 männlich und 47 weiblich) (Statistisches Bundesamt 2020).

Marleen Gräber, M.A.

marleen.graeber@krimi.uni-heidelberg.de

$\triangle$ Barbara Horten, M.A

horten@krimi.uni-heidelberg.de

1 Institut für Kriminologie, Universität Heidelberg, Heidelberg, Deutschland
Die Suizidalität von Minderjährigen stellt ein Tabuthema in der Gesellschaft dar. Der „Bullycide“ (Suizid aufgrund von Mobbing im Internet) sollte jedoch insbesondere vor dem Hintergrund des steigenden Einflusses digitaler Medien nicht unterschätzt werden. Digitale Medien besitzen für Kinder und Jugendliche einen hohen Stellenwert und nehmen erheblich auf deren Alltagsgestaltung Einfluss. Die Nutzung von Medien zum Zweck der Kommunikation oder der Informationsgewinnung erlangt auch im negativen Sinn zunehmend Bedeutung (Back 2020, S. 61). Studien belegen einen Zusammenhang zwischen vermehrtem Medienkonsum und einer erhöhten Beteiligung an Mobbing-Aktivitäten. Die Rolle digitaler Medien im Zusammenhang mit Mobbing ist unterschiedlich und besteht nicht allein aus dem Tatort und Tatmittel des Mobbings (S. 62). So kann der Konsum von gewalttätigen Serien- und Filmdarstellungen bei Kindern und Jugendlichen u. a. zu einer emotionalen Abstumpfung führen und auf lange Sicht zu einem Risikofaktor für die Entwicklung aggressiver Verhaltensweisen, einschließlich Mobbing-Aktivitäten, werden (S. 62 ff.). AuBerdem können die dargestellten medialen Inhalte Kinder und Jugendliche zu Nachahmungen des gezeigten Verhaltens verleiten.

Insbesondere verbale, nichtphysische Aggressionen und ein hoher Konsum von Fernsehen und Filmen wiesen nach verschiedenen Studien einen Zusammenhang auf. Kuntsche et al. (2006) fanden heraus, dass der intensive Konsum von Fernsehen und Videos (über zwei Stunden täglich) zu verbaler Aggression im Zusammenhang mit Mobbing bei SchülerInnern führte. Dieser Befund wurde auch von Martins und Wilson (2012) festgestellt. Die Autoren untersuchten den Einfluss des Konsums von gewalttätigen Fernsehinhalten nichtphysischer Form, z. B. Gerüchte verbreiten und Verrat begehen, auf das Mobbing-Verhalten von GrundschülerInnen. Sie zeigten, dass der Konsum dieser Art von Gewaltdarstellungen zu sozialer Aggression führte, die die 
Schädigung des sozialen Rufs oder des Selbstvertrauens des Opfers zum Ziel hatte. Der Effekt ist geschlechterspezifisch. Da Mädchen in den Medien eher soziale Gewaltformen und Jungen eher physische Gewaltinhalte konsumieren und sich ggf. mit den in Film und Fernsehen gezeigten Tätern identifizieren, übernehmen sie deren Verhalten (Back 2020, S. 66).

Der Zusammenhang zwischen der medialen Inszenierung von Suiziden und dem daran anschließenden Anstieg der Suizidraten wird als „suicide contagion“ oder „Werther-Effekt" (Phillips 1974) bezeichnet, in Anlehnung an den Suizid der Hauptfigur des Romans (Johann Wolfgang von Goethe, Die Leiden des jungen Werthers), von dem ein Nachahmungseffekt angenommen wird (Back 2020, S. 67). Aufgrund dieses Ansteckungseffektes verfassten Tomandl et al. (2005) Richtlinien zur Mediengestaltung, die Nachahmungseffekte eindämmen sollen. Diese enthalten u.a. den Aspekt, keine Details zur Suizidhandlung zu veröffentlichen (Tomandl et al. 2005, S. 7 ff.). Ein ambivalentes Beispiel stellt die Netflix-Produktion 13 Reasons Why dar, die von einer Jugendlichen handelt, die ihren Suizid u. a. als Rache gegen andere instrumentalisiert. Zimerman et al. (2018) untersuchten die Wirkung der Serie auf Jugendliche. Hierfür befragten sie 21.062 Personen, die größtenteils weiblich und zwischen 15 und 17 Jahre alt waren und alle Folgen der Serie gesehen hatten, zu ihrem Verhalten und Empfinden. Die Autoren kamen u. a. zu dem Ergebnis, dass Jugendliche, die vor dem Konsum der Serie andere gemobbt hatten $(41 \%)$, ihr Verhalten nach dem Schauen der Serie überdacht hatten (96\%) (Zimerman et al. 2018, S. 610f.). Von diesen $96 \%$ gaben $90 \%$ an, andere nun weniger zu mobben (S. 611). Die von den Autoren ausgewiesene methodische Schwäche der Studie lag in einer Verzerrung der Stichprobe. Zum Beispiel wurden nur Jugendliche befragt, die der Serie gegenüber positiv eingestellt waren, weil ein Einschlusskriterium darin bestand, alle Folgen der Serie gesehen zu haben. Die Studie von Zimerman et al. (2018) belegte neben einer rückläufigen Tendenz zum Mobbing-Verhalten bei einigen Jugendlichen mit einer langen Vorgeschichte von Suizidgedanken eine Verringerung der Selbstmordgedanken. Es gaben 59\% der Jugendlichen, die eine lange Vorgeschichte von Suizidgedanken hatten, einen Rückgang dieser Gedanken an. Der Konsum der Serie führte bei einigen der Befragten zu einem „Papageno-Effekt“. Der „PapagenoEffekt" (Niderkrotenthaler et al. 2010) stellt das Gegenteil zum „Werther-Effekt" dar und zeigt in Anlehnung an die Figur des „Papageno“ (Wolfgang Amadeus Mozart, Die Zauberföte) u. a. Alternativen zum Suizid auf (Till und Niederkrotenthaler 2019). Somit können Medieninhalte auch eine protektive Wirkung haben. Allerdings äußerten in der Studie knappe 17\%, mehr Suizidgedanken nach dem Schauen der Serie gehabt zu haben (Zimerman et al. 2018, S. 610f.). Medienkompetenz, die etwa die Eltern ihren Kindern ver- mitteln, ist ein zentraler Schutzfaktor vor dem unreflektierten Konsum von Medien, auch um Kinder und Jugendliche davor zu bewahren, möglicherweise zum Mobbing-Täter zu werden (Back 2020, S. 71 ff.).

Mobbing unter Kindern und Jugendlichen ist kein neues Phänomen, jedoch ist durch die sich stetig weiter verbreitenden Möglichkeiten im digitalen Raum Cybermobbing als eine relativ neue Erscheinungsform auf diesem Gebiet hinzugekommen. Cybermobbing ähnelt dem Phänomen des Schulmobbings hinsichtlich der länger andauernden Schädigungsabsicht, und es können, wie auch im realen Umfeld, mehrere Personen und ganze Gruppen (z. B. FacebookGruppen) beteiligt sein (Katzer 2014, S. 60). Es existiert ein Machtungleichgewicht zwischen Täter und Opfer. Gleichwohl weist das Phänomen des Cybermobbings spezifische Merkmale auf. Diese bestehen aus einem höheren Anonymisierungsgrad der Täter im digitalen Raum und zugleich einem höheren Öffentlichkeitsgrad der Demütigungen des Opfers (S. 62). Außerdem liegt ein hohes Maß an Schutzlosigkeit vor, weil die Täter mittels Internet durch verschiedene Endgeräte immer und überall Zugriff auf das Opfer haben. Es gibt kaum Rückzugsmöglichkeiten vor MobbingAttacken, da die Täter bis in das eigentlich sichere Zuhause ihrer Opfer vordringen können.

Die Verhaltensweisen des Cybermobbings reichen von Beleidigungen, sozialem Ausschluss, direkten Bedrohungen und Erpressungen, dem Verbreiten von Lügen, Geheimnissen oder privatem Bildmaterial bis hin zu der Identitätsübernahme einer Person (Katzer 2014, S. 62). Im Juli 2020 berichtete der Bayerische Rundfunk über ein rechtskräftiges Urteil gegen einen zum Tatzeitpunkt 14-jährigen Täter, der im Jahre 2019 über sein Mobbing-Opfer gefälschte Todesanzeigen erstellte. Die Strafe bestand aus einer Woche Dauerarrest und $120 \mathrm{~h}$ gemeinnütziger Arbeit (Zacher et al. 2020). Oftmals verletzen Mobbing-Aktivitäten Betroffene direkt oder indirekt über eine längere Dauer.

Bislang existiert kein eigener Straftatbestand des Mobbings bzw. des Cybermobbings, sondern die Handlung wird unter verschiedenen Strafvorschriften eingeordnet (Schirra 2020, S. 21 f.). So handelt es sich z.B. beim Androhen eines Mordes über eine Internetplattform um den Straftatbestand der Bedrohung ( $\$ 241$ StGB) (Katzer 2014, S. 73). Für Cybermobbing können nach dem Strafgesetzbuch auBerdem folgende Tatbestände in Betracht kommen: Beleidigung ( $§ 185 \mathrm{StGB}$ ), üble Nachrede ( $\$ 186 \mathrm{StGB}$ ), Verleumdung ( $\$ 187 \mathrm{StGB})$, Verletzung der Vertraulichkeit des Wortes ( $\$ 201 \mathrm{StGB}$ ), Verletzung des höchstpersönlichen Lebensbereichs durch Bildaufnahmen ( $\$ 201 \mathrm{a}$ StGB), Nötigung ( $\$ 240 \mathrm{StGB})$ und Gewaltdarstellung ( $\$ 131 \mathrm{StGB}$ ) (S. 73). Auch das „happy slapping“ („fröhliches Schlagen"), bei dem die meist jugendlichen Täter ein oftmals willkürlich gewähltes Opfer angreifen, den Angriff filmen und diesen im Anschluss veröffentlichen, wird teils dem 
Cybermobbing zugeordnet und kann z. B. eine nach $§ 131$ StGB strafbare Gewaltdarstellung sein (Preuß 2019, S. 97). In der Fachliteratur herrschen geteilte Auffassungen, ob ein Tatbestand des Cybermobbings eingeführt werden sollte.

Insbesondere das psychische Leid, was durch die Mobbing-Attacken verursacht wird, ist für die Betroffenen u. a. aufgrund der permanenten Angriffsmöglichkeiten im digitalen Raum sehr belastend. Die psychischen Folgen von Cybermobbing reichen von kurzfristigen Belastungen (z. B. Wut, Frustration und psychosomatischen Beschwerden) über dauerhafte Belastungen (z. B. chronische Traumatisierung, Depressionen) in Einzelfällen bis zu ,school shootings“" (Amokläufen in Schulen) (Weber 2018, S. 50f.). Außerdem können Cybermobbing-Attacken zu selbstverletzendem Verhalten, Suizidversuchen und vollendetem Suizid (Bullycide) führen (Katzer 2014, S. 105 f.). Auch wenn der Suizid eine vergleichsweise seltene Folge von Cybermobbing darstellt, sind die Fälle in der Kinder- und Jugendpsychiatrie, die mit Cybermobbing in Verbindung stehen, angestiegen und werden vermutlich auch in $\mathrm{Zu}$ kunft weiteransteigen (Katzer 2014, S. 17). Faktoren, die Suizidalität begünstigen, sind neben psychischen Erkrankungen (u. a. affektive Störungen), psychosozialen Faktoren (u. a. soziale Isolation) und sexuellem Missbrauch auch soziale Stressoren, wie Mobbing-Erfahrungen sie darstellen (Weitzel 2020, S. 141).

Mehrere Studien verweisen auf einen Zusammenhang zwischen Cybermobbing und Suizidgedanken (Bonanno und Hymel 2013; Brunstein Klomek et al. 2009; Hinduja und Patchin 2010). Weitzel (2020, S. 143) nennt einen bislang nicht hinreichend berücksichtigten Richtungszusammenhang: Es ist unklar, inwieweit Mobbing zu suizidalen Gedanken führt und in welchem Ausmaß Jugendliche mit diesen Gedanken leichter zu Mobbing-Opfern werden. AuBerdem wiesen Pinto und Whisman bereits 1996 darauf hin, dass Jugendliche mit Suizidgedanken aufgrund kognitiver Verzerrungen Handlungen anderer leichter als Mobbing auffassen könnten (S. 143).

Bislang gibt es nur wenige Längsschnittstudien über den Einfluss von Mobbing-Erfahrungen auf einen späteren Suizid. Im Jahre 2009 führten Brunstein Klomek et al. eine Langzeitstudie über den Zusammenhang zwischen Mobbing-Erfahrungen im Alter von 8 Jahren und späterem Suizidversuch bzw. vollendetem Suizid im Erwachsenenalter mit 5302 finnischen Kindern durch. Die Forscher fanden heraus, dass nur bei Mädchen ein signifikanter Zusammenhang zu späteren Selbsttötungen bestand, bei Jungen trat dieser Zusammenhang nach der Variablenkontrolle von Verhaltensproblemen (z.B. Aggression) und Depressionssymptomen (z. B. Traurigkeit) nicht auf (Brunstein Klomek et al. 2009).

In der Mobbing-Forschung gibt es weitere Themengebiete, wie etwa die Rolle der „,bystander“ (engl.: „Dabeiste- hende“, also Zeugen, die nicht eingreifen), die insbesondere bei Mobbing im digitalen Raum eine Rolle spielen (Weitzel 2020, S. 143). Diese sind bis heute kaum Gegenstand der Forschung. Weitzel (2019) fand in einer Untersuchung heraus, dass das tatenlose Beobachten von Cybermobbing auch bei Außenstehenden, die nicht von Mobbing betroffen waren, zu einem starken Prädiktor für suizidale Gedanken zählte (S. 143). Das Gefühl von Hoffnungslosigkeit und der wahrgenommenen sozialen Unterstützung nur eines engen Freundes sind Faktoren, die sich auf die Suizidalität von Jugendlichen mit Mobbing-Erfahrungen negativ auswirken können (S. 144).

Das Bündnis gegen Cybermobbing, ein Verein, der es sich zur Aufgabe gemacht hat, der Entwicklung von Mobbing in der digitalen Welt entgegenzuwirken, veröffentlichte 2013 und 2017 die Ergebnisse von Schüler-, Eltern- und Lehrerbefragungen zum Thema Cybermobbing. Im Jahre 2016 wurden 1586 Datensätze von SchülerInnen im Alter von 10 bis 21 Jahren erhoben (Bündnis gegen Cybermobbing 2017, S. 14). Nahezu $13 \%$ der befragten SchülerInnen gaben an, von Cybermobbing betroffen zu sein (S. 81). Die Autoren der Studie verweisen darauf, dass das Mobbing an Schulen zugenommen habe und durch die feste Verankerung etwa der Smartphones im Alltag nicht strikt vom Schulmobbing getrennt werden könne. Die Studie ergab, dass an Gesamtschulen und Gymnasien die wenigsten Fälle von Cybermobbing vorlagen (10-11\%), während Berufsschulen die meisten Fälle verzeichneten (26\%). Mädchen waren häufiger Opfer von Cybermobbing. Die betroffenen Jugendlichen wurden am häufigsten online beschimpft oder beleidigt (72\%), knapp die Hälfte litt unter der Verbreitung von Lügen oder Gerüchten, und knapp jeder 4. Jugendliche wurde unter Druck gesetzt, erpresst oder bedroht (S. 82). Für das Cybermobbing wurden am häufigsten MessagingDienste benutzt und am zweithäufigsten soziale Netzwerke. Es erfolgten $78 \%$ der Angriffe mittels ,instant messanging“ (z.B. WhatsApp), dies entsprach einer Zunahme um $49 \%$ im Vergleich zur Vorgängerstudie 2013 (S. 83). Hier war also eine starke Verschiebung des Tatmittels für die Angriffe durch die Täter zu verzeichnen. Es gaben $20 \%$ der Täter an, selbst schon einmal Opfer von Cybermobbing gewesen zu sein (S. 84). Die Gründe der Täter bezeichneten die Autoren größtenteils als reaktiv: Bezogen auf die Täter erklärten $45 \%$ ihre Handlungen damit, dass die Person die Attacke verdient habe, und weitere $43 \%$, dass sie Ärger mit dem Mobbing-Opfer gehabt hätten. An 3. Stelle nannten $28 \%$ als Grund, selbst von der Person gemobbt worden zu sein (S. 85). Die Folgen für die Betroffenen reichten von dem Gefühl, sich verletzt zu fühlen (62\%), über Wut (47\%) und Angst (36\%) bis zu Suizidgedanken bei jedem 5. MobbingOpfer (S. 86).

Weber (2018) untersuchte im Stadtgebiet Bonn Cybermobbing unter SchülerInnen in der Sekundarstufe I 
(Weber 2018, S. 131). Es wurden 2113 schriftliche Fragebogen, die SchülerInnen im Schuljahr 2014 zum Thema Nutzungsverhalten von Internet und Handy sowie Cybermobbing-Erfahrungen ausgefüllt hatten, ausgewertet (S. $137 \mathrm{ff}$.) Von den SchülerInnen gaben $45 \%$ an, mindestens eine Erfahrung mit Cybermobbing gemacht zu haben, am häufigsten waren dies Beleidigungen oder das Verbreiten von Gerüchten (S. 166). Bei den Reaktionen und Folgen war mit $36 \%$ bei den betroffenen SchülerInnen am häufigsten, ihr schwerwiegendstes CybermobbingErlebnis als „nicht so schlimm“ empfunden zu haben, 9\% der befragten SchülerInnen gaben allerdings an, nach dem schlimmsten Vorfall nicht mehr leben zu wollen (S. 184). Weber verweist darauf, dass dies einerseits auf tatsächlich schwerwiegende Cybermobbing-Attacken hindeutet, andererseits aber in einigen Fällen eine für Jugendliche typische Überreaktion stattgefunden hat. Die relative hohe Zahl spricht dafür, dass Cybermobbing einen starken Effekt auf Kinder und Jugendliche haben kann. Schulen sollten sich deshalb gezielt mit der Problematik auseinandersetzen und das Thema etwa in Form von Projekttagen oder Unterrichtsreihen in den Schulalltag einbauen (S. 305).

Die digitale Welt ist, so zeigt sich, ein Tatort für Mobbing, wobei die Trennlinien zwischen Mobbing in der realen und der virtuellen Welt, auch wegen der aktuellen Situation in den Schulen, wohl auch in Zukunft noch weiter verschwimmen werden.

Funding Open Access funding provided by Projekt DEAL.

Interessenkonflikt M. Gräber und B. Horten geben an, dass kein Interessenkonflikt besteht.

Open Access Dieser Artikel wird unter der Creative Commons Namensnennung 4.0 International Lizenz veröffentlicht, welche die Nutzung, Vervielfältigung, Bearbeitung, Verbreitung und Wiedergabe in jeglichem Medium und Format erlaubt, sofern Sie den/die ursprünglichen Autor(en) und die Quelle ordnungsgemäß nennen, einen Link zur Creative Commons Lizenz beifügen und angeben, ob Änderungen vorgenommen wurden.

Die in diesem Artikel enthaltenen Bilder und sonstiges Drittmaterial unterliegen ebenfalls der genannten Creative Commons Lizenz, sofern sich aus der Abbildungslegende nichts anderes ergibt. Sofern das betreffende Material nicht unter der genannten Creative Commons Lizenz steht und die betreffende Handlung nicht nach gesetzlichen Vorschriften erlaubt ist, ist für die oben aufgeführten Weiterverwendungen des Materials die Einwilligung des jeweiligen Rechteinhabers einzuholen.

Weitere Details zur Lizenz entnehmen Sie bitte der Lizenzinformation auf http://creativecommons.org/licenses/by/4.0/deed.de.

\section{Literatur}

Back SJ (2020) Exkurs: Mediale Inszenierung von Mobbing - Zusammenhänge zwischen jugendlichem Medienkonsum, Mobbing und Suizid. In: Böhmer M, Georges S (Hrsg) Mobbing an Schulen. Maßnahmen zur Prävention, Intervention und Nachsorge. Springer, Berlin, Heidelberg, S 57-77
Bonanno RA, Hymel S (2013) Cyber bullying and internalizing difficulties: Above and beyond the impact of traditional forms of bullying. J Youth Adolescence 42:685-697

Brunstein Klomek AB, Sourander A, Niemelä S, Kumpulainen K, Piha J, Tamminen T, Almqvist F, Gould M (2009) Childhood bullying behaviors as a risk for suicide attempts and completed suicides: a population-based birth cohort study. J Am Acad Child Adolesc Psychiatry 48:254-261

Bündnis gegen Cybermobbing (2017) Cyberlife II Spannungsfeld zwischen Faszination und Gefahr Cybermobbing bei Schülerinnen und Schülern. Zweite empirische Bestandsaufnahme bei Eltern, Lehrkräften und Schülern/ innen in Deutschland (Folgestudie von 2013). https://www.buendnis-gegen-cybermobbing. de/fileadmin/pdf/studien/2016_05_02_Cybermobbing_2017End. pdf. Zugegriffen: 23. Juli 2020

Centers for Disease Control and Prevention (2011) Leading causes of death by age group. http://www.cdc.gov/injury/wisqars/ pdf/leading_causes_of_death_by_age_group_2011-a.pdf. Zugegriffen: 14. September 2020

Hinduja S, Patchin JW (2010) Bullying, cyberbullying, and suicide. Arch Suicide Res 14:206-221. https://doi.org/10.1080/ 13811118.2010 .494133

Katzer C (2014) Cybermobbing - Wenn das Internet zur W@ffe wird. Springer, Berlin, Heidelberg

Kuntsche E, Pickett W, Overpeck M, Craig W, Boyce W, de Matos MG (2006) Television viewing and forms of bullying among adolescents from eight countries. J Adolesc Health 39:908-915

Niederkrotenthaler T, Voracek M, Herberth A, Till B, Strauss M, Etzersdorfer E, Eisenwort B, Sonneck G (2010). Role of media reports in completed and prevented suicide: Werther v. Papageno effects. Brit J Psychiatry 197:234-243

Martins N, Wilson BJ (2012) Social aggression on television and its relationship to children's aggression in the classroom. Hum Commun Res 38:48-71

Phillips DP (1974) The influence of suggestion on suicide: Substantive and theoretical implications of the Werther effect. Am Sociol Rev 39:340-354. https://doi.org/10.2307/2094294

Preuß T (2019) Erforderlichkeit der Kriminalisierung des Cybermobbings - Sinnvolle Schließung einer Gesetzeslücke oder bloßes Symbolstrafrecht? In: Kriminalpolitische Zeitschrift 2/ 2019. https://kripoz.de/2019/03/07/erforderlichkeit-der-kriminalisie rung-des-cybermobbings-sinnvolle-schliessung-einer-gesetzeslue cke-oder-blosses-symbolstrafrecht/. Zugegriffen: 23. Juli 2020

Radeloff D, Lempp T (2012) Suizide im Kindes- und Jugendalter. Eine 13-Jahreserhebung im Einzugsgebiet einer deutschen Großstadt. Z Kinder Jugendpsychiatr Psychother 40:263-269. https:// doi.org/10.1024/1422-4917/a000179

Schirra HJ (2020) Exkurs: „Mobbing als Straftat“ - Betrachtung der aktuellen rechtlichen Einordnung von Mobbing in Deutschland. In: Böhmer M, Georges S (Hrsg) Mobbing an Schulen. Maßnahmen zur Prävention, Intervention und Nachsorge. Springer, Berlin, Heidelberg, S 19-31

Statistisches Bundesamt (Destatis) (2020) Todesursachenstatistik. Deutschland. Gestorbene (Anzahl). DeStatis, Wiesbaden

Smith PK, Mahdavi J, Carvalho M, Fisher S, Russell S, Tippett N (2008) Cyberbullying: its nature and impact in secondary school pupils. J Child Psychol Psychiatr 49:376-385. https://doi.org/10. 1111/j.1469-7610.2007.01846.x

Till B, Niederkrotenthaler T (2019) Medien und Suizid: der aktuelle Forschungsstand zum Werther- und Papageno-Effekt - eine Übersichtsarbeit. Psychother Forum 23:120-128

Tomandl G, Sonneck G, Stein C, Niederkrotenthaler T (2005) Leitfaden zur Berichterstattung über Suizid. Kriseninterventionszentrum. https://www.iasp.info/pdf/task_forces/Austria_Leitfaden_ Berichterstattung.pdf. Zugegriffen: 23. Juli 2020

Weber J (2018) Cybermobbing - wenn neue Medien fertigmachen. Eine Untersuchung zum Cybermobbing im Stadtgebiet Bonn. LIT, Berlin 
Weitzel L (2019) Bullying involvement, suicidal ideation, and the role of perceived social support. University of Luxembourg, Luxembourg

Weitzel L (2020) Exkurs: Suizidalität und Mobbing. In: Böhmer M, Georges S (Hrsg) Mobbing an Schulen. Maßnahmen zur Prävention, Intervention und Nachsorge. Springer, Berlin, Heidelberg, S $131-150$

Zacher J, Bader W, Kubina M (2020) Mobbing mit falschen Todesanzeigen: Schüler verurteilt. In: Bayerischer Rundfunk. https:// www.br.de/nachrichten/bayern/gefaelschte-todesanzeigen-schue ler-zu-arrest-verurteilt,S5Mo9qr. Zugegriffen: 29. Juli 2020

Zimerman A, Caye A, Salum GA, Passos IC, Kieling C (2018) Revisiting the werther effect in the 21st century: bullying and suicidality among adolescents who watched 13 reasons why. J Am Acad Child Adolesc Psychiatry 57(7):610-613. https://doi.org/10.1016/ j.jaac.2018.02.019 